\title{
Positive Feedback Hypothesis on Development of Essential Hypertension
}

\author{
Hiroyuki Suga, M.D., D.M.Sc.
}

\begin{abstract}
SUMMARY
A hypothesis is presented on a possible mechanism of development of the essential hypertension. The present theoretical consideration with Laplace's law and Poiseuille's law indicates that arteriolar constriction increases, unless the blood flow is reduced, both arterial blood pressure and vascular circumferential wall tension which is considered a trigger of medial hypertrophy of the constricted arteriole. If the medial hypertrophy aggravates the vascular constriction, it all the more increases arterial pressure and wall tension. Therefore, the initial arteriolar constriction, however slight, may progressively produce hypertension and augment medial hypertrophy by such a positive feedback mechanism.
\end{abstract}

\section{Additional Indexing Words:}

Primary hypertension Vascular adaptation Autoregulation Vasoconstriction Hypertensive state Vascular smooth muscle Laplace's law

\begin{abstract}
ATERIOLAR walls show medial hypertrophy at an established stage of hypertension. ${ }^{1), 2}$ Their cause and effect relation during the development of hypertension, however, remains to be elucidated. ${ }^{1,8}$ )

In this paper, a hypothesis on the cause and effect of hypertension and arteriolar hypertrophy was proposed from a theoretical consideration. The hypothesis is that initial constriction of arterioles, unless the blood flow through them is reduced, always accompanies increases in arterial blood pressure and vascular wall tension, the latter of which is considered a cause of vascular medial hypertrophy. If the hypertrophy increases the constriction, it progressively aggravates hypertension and arteriolar hypertrophy.
\end{abstract}

\section{Methods AND Results}

An arteriolar segment of an arbitrary length is modeled for simplicity as a cylindrical tube of inner radius $r_{i}$ and wall thickness $d$. Cross sectional area of

From the Department of Physiology, Faculty of Medicine, University of Tokyo, Tokyo 113.

Present address: Department of Biomedical Engineering, The Johns Hopkins University School of Medicine, Baltimore, Maryland 21205, U.S.A.

Received for publication June 3, 1975. 
the wall, $\mathrm{S}$, is therefore given geometrically as

$$
S=\pi\left(d^{2}+2 r_{i} d\right)
$$

which is maintained constant during constriction or dilatation unless medial hypertrophy occurs. ${ }^{3}$ ) Blood flow resistance through the segment, $R$, is formulated from Poiseuille's law as

$$
\mathrm{R}=\mathrm{Kr}_{\mathrm{i}}^{-4}
$$

where $\mathrm{K}$ is a coefficient given by the length of the tube and viscosity of the blood, being assumed to be a constant. Upstream pressure in the segment, $P$, and the flow through it, I, are interrelated as

$$
P=I R
$$

where downstream pressure is assumed to be atmospheric, or zero.

Circumferential wall tension per unit length of the segment at the upstream end, $T$, should be in equilibrium with internal pressure $\mathbf{P}$ and external pressure Pe. ${ }^{4)}$ Therefore,

$$
\mathrm{T}=\mathrm{Pr}_{\mathrm{i}}-\mathrm{Pe}\left(\mathrm{r}_{\mathrm{i}}+\mathrm{d}\right)
$$

Assuming $\mathrm{Pe}=0$, this equation is reduced to

$$
\mathrm{T}=\operatorname{Pr}_{\mathrm{i}}
$$

Dividing $\mathrm{T}$ with wall thickness $\mathrm{d}, \mathrm{T} / \mathrm{d}$, yields average wall tension per unit cross sectional area, or stress, which is given as

$$
\mathrm{T} / \mathrm{d}=\operatorname{Pr}_{\mathfrak{i}} \mathrm{d}^{-1}
$$

Wall thickness $d$ is a function of $r_{1}$ and $S$. Solving Eq. 1 for $d$ yields

$$
\mathrm{d}=\left(\mathrm{r}_{\mathrm{t}}^{2}+\mathrm{S} / \pi\right)^{1 / 2}-\mathrm{r}_{\mathrm{i}}
$$

Therefore, when all $\mathrm{S}, \mathrm{I}$, and $\mathrm{K}$ values are specified, $\mathrm{T}, \mathrm{T} / \mathrm{d}, \mathrm{P}, \mathrm{R}$, and $\mathrm{d}$ can be calculated as a function of $r_{i}$. Fig. 1 shows them after being normalized to the respective control values.

It is likely to occur naturally that arterioles constrict functionally by some means (neural, humoral, myogenic) and the blood flow through them does not decrease, ${ }^{5)}$ i.e., $r_{i}$ decreases but I does not. Arterial blood pressure necessarily increases simultaneously. This is the hemodynamic state that is assumed in the present mathematical considerations.

As explicitly shown in Fig. 1 , the decrease in $r_{i}$ in the preceding condition increases $\mathrm{T}$ and $\mathrm{T} / \mathrm{d}$ as well as $\mathrm{P}, \mathrm{R}$, and $\mathrm{d}$. This suggests that circumferential wall tension and stress do increase with the initial constriction of arterioles which accompanies the increase in arterial blood pressure. If the blood flow rather increases with arteriolar constriction as in a hyperkinetic state, ${ }^{6)}$ the increase in wall tension is enhanced by so much. One might think that arteriolar wall tension and stress

* Although Eq. 4 appears the same with $T=$ Pr from Laplace's law for a thin wall tube, it was derived from a thick wall tube. They take the same formula only when external pressure is zero. 


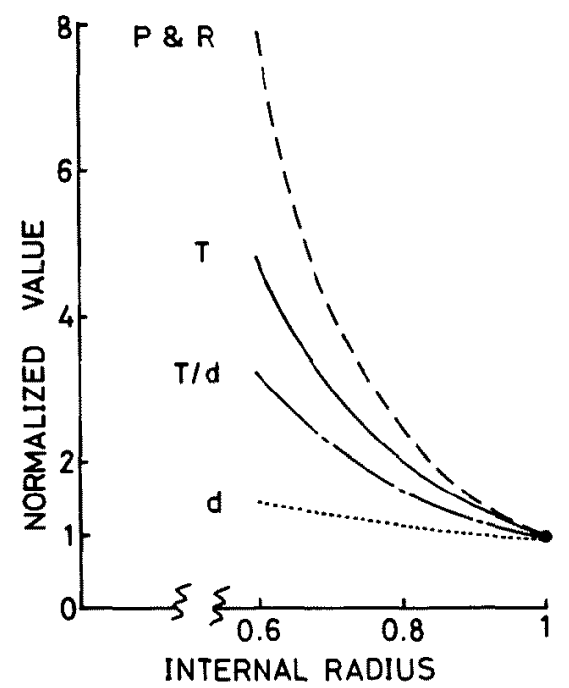

Fig. 1. Relationships of arteriolar wall circumferential tension (T), tension per unit wall thickness or stress $(T / d)$, arterial blood pressure $(P)$, flow resistance ( $R$ ), and wall thickness (d) to arteriolar internal radius $\left(r_{1}\right)$. All the variables are normalized so that their values at control state be unity. Blood flow through the arterioles is constant. Wall to lumen ratio, $d / r_{i}$, at control state is assumed to be 0.2 , which is a typical value for the normal arteriole according to Reference 7 .

decrease with the vascular constriction, according to Laplace's law. This holds true when arterial blood pressure is unchanged and hence blood flow is reduced. But when blood flow is unchanged with the constriction, the wall tension and stress do increase.

Although the preceding formulae are for the upstream end of the arteriolar segment, similar formulae can be given for any other point of the segment by substituting $P(1-x)$ for $P$, where $x=$ normalized distance of the point from the upstream end. Therefore, the above discussion holds for any point of the vascular segment model.

The increase in wall tension or stress can be considered to be a cause of arteriolar medial hypertrophy as the adaptation of smooth muscles to overload. ${ }^{1-3}{ }^{\text {) }}$ Therefore, the initial arteriolar constriction will produce arteriolar hypertrophy.

It is extremely important whether the vascular hypertrophy is of a constrictive type or a dilating type. The type of hypertrophy depends on whether smooth muscle layers grow the thickness more or the length more.

If the hypertrophy is constrictive, $r_{i}$ will be reduced further. When blood flow remains the same, arterial pressure will be increased and wall tension and stress will be further increased. This process is a vicious cycle by a positive feedback. Clinical and experimental findings on the vascular wall to lumen ratio in hypertensive subjects indicate evidence of the constrictive type of medial hypertrophy. ${ }^{11,2)}$

If the hypertrophy is dilating, the initial constriction of arterioles will be can- 
celled by the dilatation and arterial blood pressure will be back to normal. The process is a negative feedback.

\section{Discussion}

That the different types of medial hypertrophy cause the different responses of blood pressure is interesting in relation to the hereditary predisposition of hypertension. ${ }^{1}$ This study suggests the necessity of investigation how vascular smooth muscles adapt their size and number to the overload.

The assumption of maintained blood flow under vasoconstriction is reasonable since literature ${ }^{1,6)}$ shows either unchanged or rather increased blood flow during the initial development of hypertension.

In this analysis, it was assumed that arterioles constrict primarily. The situation is valid also when arterial blood pressure increases first with higher cardiac output and arterioles constrict secondarily due to the autoregulatory mechanism.

The present theoretical study provides, although speculative, a hypothesis on the development of essential hypertension. The key point of the mechanism is how vascular smooth muscles adapt to the overloaded tension.

\section{REFERENCES}

1. Folkow B, Hallbäck M, Lundgren $Y$, Sivertsson R: Importance of adaptive changes in vascular design for establishment of primary hypertension, studied in man and in spontaneously hypertensive rats. Circulat Res 32 (Suppl I): 2, 1973

2. Suwa N, Takahashi T: Morphological and Morphometrical Analysis of Circulation in Hypertension and Ischemic Kidney. Urban \& Schwarzenberg, München-Berlin-Wien, 1971

3. Wagenvoort CA: Vasoconstriction and medial hypertrophy in pulmonary hypertension. Circulation 22: 553, 1960

4. Fung YC: Biomechanics. Its scope, history and some problems of continuum mechanics in physiology. Appl Mech Rev 21: 1, 1968

5. Freis ED: Hemodynamics of hypertension. Physiol Rev 40: 27, 1960

6. Pfeffer MA, Frohlich ED: Hemodynamic and myocardial function in young and old normotensive and spontaneously hypertensive rats. Circulat Res 32 (Suppl I): 28, 1973

7. Burton AC: Physical principles of circulatory phenomena: the physical equilibria of the heart and blood vessels. In Handbook of Physiology, 2, Girculation, I, pp 85-106, 1962

8. Page IH: Arterial hypertension in retrospect. Circulat Res 34: 133, 1974 